\title{
Truncation Errors in Padé Approximations to Certain Functions: An Alternative Approach
}

\author{
By David Elliott
}

1. Introduction. The problem of finding rational approximations to functions has received a considerable amount of attention recently, and many methods exist for finding such approximations. A brief survey of the most widely used methods has been given by Cheney and Southard [1]. In all problems of approximation, it is essential to know the truncation error which arises when the function is replaced by its approximation. Ideally, one would also like to have a realistic a priori estimate of this error. However such estimates are not in general easy to obtain. For example, in the case of polynomial approximations to a function, the truncation error can frequently be expressed in terms of a higher derivative of the function at some indeterminate point. Even in cases where the $n$th derivative of a function may be readily obtained, an estimate of an upper bound of the truncation error obtained by considering upper bounds of the higher-order derivatives is frequently much larger than the actual truncation error. Consequently such an estimate is not very useful for making an a priori estimate of the degree of the approximation to be used. For polynomial approximations, realistic estimates for the truncation error can sometimes be found by first of all expressing the truncation error as a contour integral, and then making use of asymptotic methods to evaluate this integral. It has been observed that if we approximate a function $f(x)$ by a polynomial $p_{n}(x)$ of degree $n$, then an asymptotic estimate of this contour integral form of the truncation error for large $n$, frequently gives a good estimate of the error even for small values of $n$. In this paper we shall show that a similar approach can also be used for certain rational approximations, to give excellent a priori estimates.

Suppose that we wish to approximate a given function $f(x)$ by means of a rational approximation $p_{m}(x) / q_{n}(x)$, where $p_{m}(x)$ and $q_{n}(x)$ are polynomials of degree $m$ and $n$ respectively. In this paper we shall consider only the so-called Padé approximations to $f(x)$. These approximations are such that if we consider the expansions of $f(x), p_{m}(x)$ and $q_{n}(x)$ about $x=0$, then

$$
f(x) q_{n}(x)-p_{m}(x)=\left(x^{m+n+1}\right),
$$

where $\left(x^{k}\right)$ denotes a power series beginning with the term in $x^{k}$. An excellent introduction to these approximations has been given by Wall [2]. In particular, we shall be interested in the main diagonal elements of the Padé table where $m=n$. For certain functions these particular rational approximations may be obtained as the odd convergents of their continued-fraction representation. These examples have also been discussed by Wall [2], but his main concern has been with the regions of convergence of the continued-fraction representations and not directly with estimates of the truncation errors of the convergents.

Received November 15, 1966. 
Following the observations of the first paragraph, we shall first obtain an expression for the truncation error $R_{m, n}(x)$ where

$$
R_{m, n}(x)=f(x)-p_{m}(x) / q_{n}(x),
$$

in the form of a contour integral. For certain hypergeometric and confluent hypergeometric functions we shall then find explicit forms of $R_{n, n}(x)$, and consider the behaviour of these for large $n$.

Such results have been previously given by Luke [3], who has amply demonstrated the usefulness of asymptotic techniques for giving excellent a priori estimates of the truncation error. Although the estimates given in this and Luke's papers are the same, the methods of development are quite different. Luke's starting point was some results previously obtained by Laguerre [4], who showed that for functions satisfying certain linear first-order differential equations, one can derive differential and difference equations for both the denominator polynomials $q_{n}(x)$, and also the function $q_{n}(x) f(x)-p_{m}(x)$. In this paper we have used a rather more direct approach which has the advantage of showing under what conditions an estimate of the error may be made, and also leads directly to the evaluation of this error.

2. An Expression For The Truncation Error. In this section we shall derive an expression for the truncation error $R_{m, n}(x)$ of Padé approximations to a function $f(x)$ in terms of a contour integral. There is no need to restrict the argument $x$ to being a real value, and we shall consider the complex value $z=x+i y$. Let us suppose that $f(t)$ is defined in the complex $t$-plane, and that $t=z$ is a particular point in this plane. In the subsequent analysis we shall consider a contour $\mathcal{C}$ in the $t$-plane which satisfies the following conditions:

(i) the points $t=0$ and $z$ are inside the contour $\mathrm{e}$,

(ii) the function $f(t)$ is holomorphic on and within $\mathfrak{e}$.

First let us consider the Taylor series expansion for the function $f(t)$ about the point $t=0$. We have (see Whittaker and Watson [5]), that at the point $t=z$,

$$
f(z)=J_{n}(z)+\frac{z^{n+1}}{2 \pi i} \int_{\mathcal{C}} \frac{f(t) d t}{(t-z) t^{n+1}},
$$

where $\Im_{n}(z)$ is a polynomial of degree $\leqslant n$. Next, for our Padé approximation we have

$$
f(z)=\frac{p_{m}(z)}{q_{n}(z)}+R_{m, n}(z)
$$

which in terms of our previous notation we can write as

$$
q_{n}(z) R_{m, n}(z) \equiv q_{n}(z) f(z)-p_{m}(z)=\left(z^{m+n+1}\right) .
$$

Equation (2.3) shows that the problem of finding $R_{m, n}(z)$ is equivalent to that of finding the truncation error of a polynomial approximation to the function $q_{n}(z) f(z)$, of degree $\leqslant m+n$. From Eq. (2.1) we have immediately that

$$
R_{m, n}(z)=\frac{z^{m+n+1}}{q_{n}(z)} \cdot \frac{1}{2 \pi i} \int_{\mathcal{C}} \frac{q_{n}(t) f(t) d t}{(t-z) t^{m+n+1}},
$$


which is the required form for the remainder. It may be noted here that equation (2.3) also gives immediately the algorithm for determining the polynomials $p_{m}(z)$ and $q_{n}(z)$. If $C_{k}(g)$ denotes the coefficient of $z^{k}$ in the power series expansion of a function $g(z)$ then the algorithm is given by

$$
\begin{aligned}
& C_{k}\left[q_{n}(z) f(z)\right]=C_{k}\left[p_{m}(z)\right] \text { for } k=O(1) m ; \\
& C_{k}\left[q_{n}(z) f(z)\right]=0 \text { for } k=(m+1)(1)(m+n) .
\end{aligned}
$$

These $(m+n+1)$ conditions are sufficient to determine the $(m+n+2)$ coefficients of $p_{m}(z)$ and $q_{n}(z)$; the further condition may be arbitrarily prescribed since we are considering the ratio of two polynomials. One frequently chooses the polynomial $q_{n}(z)$ so that $q_{n}(0)=1$.

From Eq. (2.4) we see that an a priori estimate of the truncation error $R_{m, n}(z)$ may only be obtained in those cases where we know the explicit form of the polynomial $q_{n}(z)$. For certain hypergeometric functions, it is known that the denominator polynomials for the main diagonal Padé approximations of the form $p_{n}(z) / q_{n}(z)$ and the off-diagonal approximations of the form $p_{n-1}(z) / q_{n}(z)$ can be expressed in terms of Jacobi polynomials. For certain limiting forms of the hypergeometric function, the denominator polynomials are also given in terms of the corresponding limiting forms of the Jacobi polynomials. The details of this are given in Sections 4 and 5 .

In the next three sections we shall consider the problem of evaluating the truncation error only for the rational approximations of the form $p_{n}(z) / q_{n}(z)$. Similar results for the approximations $p_{n-1}(z) / q_{n}(z)$ to these functions may be obtained in an analogous way. Since these results have also been given by Luke [3], we shall consider them no further in this paper.

3. The Hypergeometric Function ${ }_{2} F_{1}(1, \omega ; 1+\lambda ; z)$. We shall begin our analysis by considering the function ${ }_{2} F_{1}(1, \omega ; 1+\lambda ; z)$, and we shall assume throughout that both $\omega$ and $(\lambda-\omega)$ are greater than -1 . For this function we can obtain the truncation error $R_{n, n}(z)$ explicitly as the ratio of two hypergeometric functions, whence we can obtain an estimate of $R_{n, n}(z)$ for large $n$ in terms of elementary functions. Furthermore, from the explicit representation of the truncation error in this case we can obtain the truncation errors for two further functions $\Phi(1 ; 1+\lambda ; z)$ and $z^{-1} \Psi\left(1 ; 2-\omega ; z^{-1}\right)$. These are discussed in Sections 4 and 5 respectively.

The starting point of our analysis is the known continued-fraction representation for the function ${ }_{2} F_{1}(1, \omega ; 1+\lambda ; z)$. This is given by (see Wall [2]),

$$
{ }_{2} F_{1}(1, \omega ; 1+\lambda ; z)=\frac{1}{1+} \frac{b_{1} z}{1+} \frac{b_{2} z}{1+} \frac{b_{3} z}{1+} \frac{b_{4} z}{1+} \cdots,
$$

where the coefficients $b_{k}$ are defined by

$$
b_{2 k+1}=\frac{(k+\omega)(k+\lambda)}{(2 k+\lambda)(2 k+\lambda+1)} \quad \text { and } \quad b_{2 k+2}=\frac{(k+1)(k+\lambda-\omega+1)}{(2 k+\lambda+1)(2 k+\lambda+2)} .
$$

The continued-fraction representation, Eq. (3.1), converges for all values of $z$ in the complex $z$-plane cut along the positive real axis from 1 to $\infty$. From the odd convergents of this continued fraction we obtain the rational approximations $p_{n}(z) / q_{n}(z)$ with $p_{n}(0) \neq 0$, where the denominator polynomials $q_{n}(z)$ are given by (see Wall $[2$, 
Eq. (89.20)]),

$$
q_{n}(z)=\frac{\Gamma(n+\lambda+1) \Gamma(n+1)}{\Gamma(2 n+\lambda+1)}(-z)^{n} P_{n}^{(\omega, \lambda-\omega)}\left(1-2 z^{-1}\right) .
$$

The polynomial $P_{n}^{(\alpha, \beta)}(x)$ denotes the Jacobi polynomial. From Eq. (2.4) we can write the remainder $R_{n, n}(z)$ for this function in the form,

$$
\begin{aligned}
& R_{n, n}(z)=\frac{z^{2 n+1} I(z)}{(-z)^{n} P_{n}{ }^{(\omega, \lambda-\omega)}\left(1-2 z^{-1}\right)}, \\
& \quad \text { where } \quad I(z)=\frac{1}{2 \pi i} \int_{\mathfrak{C}} \frac{(-t)^{n} P_{n}{ }^{(\omega, \lambda-\omega)}\left(1-2 t^{-1}\right)_{2} F_{1}(1, \omega ; 1+\lambda ; t)}{(t-z) t^{2 n+1}} d t .
\end{aligned}
$$

In order to evaluate the function $I(z)$, we choose the contour $\mathcal{C}$ as follows: (i) a line $A B$ just above and parallel to the positive real axis from $t=1$ (the point $A$ ) to $t=\rho$ (the point $B$ ), where $\rho$ is large, (ii) a large circle of radius $\rho$, centre at the origin, from the point $B$ to the point $C\left(t=\rho e^{2 \pi i}\right)$, (iii) a line $C D$ just below and parallel to the positive real axis from $C$ to the point $D\left(t=1 . e^{2 \pi i}\right)$, and finally (iv) a small circle of radius $r$, centre at $t=1$ from $D$ to $A$. The contour $\mathcal{C}$ is described positively in the sense $A B C D A$ as given above. It is not difficult to show that the contributions to $I(z)$ from the circles of radii $r$ and $\rho$, tend to zero in the limits as $r \rightarrow 0$ and $\rho \rightarrow \infty$.

Along $A B$, we can write $t=(u+1) e^{i 0}$ and along $C D, t=(u+1) e^{2 \pi i}$. On taking the limits as $r \rightarrow 0$ and $\rho \rightarrow \infty$, we find

$$
\begin{aligned}
I(z)= & \frac{(-1)^{n}}{2 \pi i} \int_{0}^{\infty} \frac{P_{n}{ }^{(\omega, \lambda-\omega)}\left(\frac{u-1}{u+1}\right)}{(u+1-z)(u+1)^{n+1}} \\
& \times\left\{{ }_{2} F_{1}\left(1, \omega ; 1+\lambda ;(u+1) e^{i 0}\right)-{ }_{2} F_{1}\left(1, \omega ; 1+\lambda ;(u+1) e^{2 \pi i}\right)\right\} d u .
\end{aligned}
$$

From the continuation formulae for the hypergeometric function (Erdélyi [6, p. 63, Eq. (17)]), we have, after some simplification, that

$$
\begin{gathered}
{ }_{2} F_{1}\left(1, \omega ; 1+\lambda ;(u+1) e^{i 0}\right)-{ }_{2} F_{1}\left(1, \omega ; 1+\lambda ;(u+1) e^{2 \pi i}\right) \\
=\frac{2 \pi i \Gamma(\lambda+1)}{\Gamma(\lambda-\omega+1) \Gamma(\omega)}\left(\frac{u}{u+1}\right)^{\lambda-\omega} \frac{1}{(u+1)^{\omega}} .
\end{gathered}
$$

From equations (3.5) and (3.6) we have,

$$
I(z)=\frac{\Gamma(\lambda+1)}{\Gamma(\lambda-\omega+1) \Gamma(\omega)} J(z) .
$$

$$
\text { where } J(z)=\int_{0}^{\infty}\left(\frac{u}{u+1}\right)^{\lambda-\omega} \frac{P_{n}^{(\lambda-\omega, \omega)}\left(\frac{1-u}{1+u}\right)}{(u+1-z)(u+1)^{n+\omega+1}} d u \text {. }
$$

Here we have made use of the relation that $P_{n}^{(\alpha, \beta)}(x)=(-1)^{n} P_{n}^{(\beta, \alpha)}(-x)$. Finally, in order to evaluate the integral $J(z)$, we make a further change of the variable of integration from $u$ to $v$, where $v=(1-u) /(1+u)$, so that $-1 \leq v \leq 1$. On using Rodrigues' definition for the Jacobi polynomial, we have 


$$
J(z)=\frac{(-1)^{n}}{\Gamma(n+1) 2^{2 n+\lambda}} \int_{-1}^{1} \frac{(1+v)^{n}}{[2-z(1+v)]} \frac{d^{n}}{d v^{n}}\left[(1-v)^{n+\lambda-\omega}(1+v)^{n+\omega}\right] d v .
$$

On integrating $n$ times by parts and making use of the relation

$$
\frac{d^{n}}{d v^{n}}\left[\frac{(1+v)^{n}}{2-z(1+v)}\right]=\frac{2^{n} \Gamma(n+1)}{[2-z(1+v)]^{n+1}},
$$

which may be readily proved by induction, we find that

$$
J(z)=\frac{1}{2^{n+\lambda}} \int_{-1}^{1} \frac{(1-v)^{n+\lambda-\omega}(1+v)^{n+\omega}}{[2-z(1+v)]^{n+1}} d v .
$$

If we make a further change of the variable of integration from $v$ to $(1+v) / 2$, and use the definition of the hypergeometric function as given in $[6$, p. 114, Eq. 1], we find that

$$
\begin{aligned}
J(z)= & \frac{\Gamma(n+\lambda-\omega+1) \Gamma(n+\omega+1)}{\Gamma(2 n+\lambda+2)} \\
& \times{ }_{2} F_{1}(n+1, n+\omega+1 ; 2 n+\lambda+2 ; z)
\end{aligned}
$$

for all $n=0,1,2, \cdots$, provided $|\arg (1-z)|<\pi$.

From Eqs. (3.11), (3.7) and (3.4), if we also represent $P_{n}^{(\omega, \lambda-\omega)}\left(1-2 z^{-1}\right)$ as a hypergeometric function (see Szegö [8, Eq. (4.21.2)]), we have the required expression for the remainder $R_{n, n}(z)$;

$$
\begin{aligned}
R_{n, n}(z)= & \frac{(-1)^{n} \omega \Gamma(\lambda+1) \Gamma(n+1) \Gamma(n+\lambda-\omega+1)}{\Gamma(2 n+\lambda+2) \Gamma(\lambda-\omega+1)} z^{n+1} \\
& \times \frac{{ }_{2} F_{1}(n+1, n+\omega+1 ; 2 n+\lambda+2 ; z)}{{ }_{2} F_{1}\left(-n, n+\lambda+1 ; \omega+1 ; z^{-1}\right)} .
\end{aligned}
$$

This result as it stands is not very convenient for numerical work. However, if we assume that $n$ is large, then we may obtain an asymptotic estimate for $R_{n, n}(z)$ in terms of elementary functions. From the asymptotic forms of the hypergeometric function ${ }_{2} F_{1}(a, b ; c ; z)$ for large $a, b$ and $c$ [6, p. 77, Eqs. (16) and (17)], we find, after some algebra that,

$$
\begin{aligned}
& R_{n, n}(z)=\frac{2 \pi \Gamma(\lambda+1)}{\Gamma(\lambda-\omega+1)} \overline{\Gamma(\omega)} \cdot \frac{z^{2 n+1}(1-z)^{\lambda-\omega}}{\left\{2-z+2(1-z)^{1 / 2}\right\}^{2 n+\lambda+1}} \\
& \times\left\{1+O\left(\frac{1}{n}\right)\right\} \text {, }
\end{aligned}
$$

for $|\arg (1-z)|<\pi$. For noninteger values of $\alpha$, we define $(1-z)^{\alpha}$ to be that branch of the function which has the value +1 when $z=0$.

Eq. (3.13) has previously been obtained by Luke [3, Eq. (4.9)], although as mentioned above, his method of derivation is somewhat different from that described in this paper. However, as Luke has shown, Eq. (3.13) although obtained from equation (3.12) under the assumption that $n$ is large, does give excellent estimates of $R_{n, n}(z)$ even for small values of $n$. In the particular case of $\lambda=\omega=1$, $z=-2$, Luke has shown that equation (3.13) gives $R_{n, n}(z)$ correct to one significant figure for values of $n$ as small as 1 . Such accuracy is quite sufficient for an estimate 
of the error; moreover, for given values of $\lambda, \omega$ and $z$, the evaluation of $R_{n, n}(z)$ from Eq. (3.13) for various values of $n$ may be readily made.

4. The Confluent Hypergeometric Function $\Phi(1 ; 1+\lambda ; z)$. From the results of the previous section we can readily obtain similar results for the truncation error in the main diagonal Padé approximations of the confluent hypergeometric function $\Phi(1 ; 1+\lambda ; z)$. Luke [3] discusses this case by repeating his analysis from the same starting point as the hypergeometric function ${ }_{2} F_{1}(1, \omega ; 1+\lambda ; z)$. However, we shall derive the results of this section directly from Eq. (3.12). It is well known that if, in the hypergeometric function ${ }_{2} F_{1}(a, b ; c ; z)$, we replace $z$ by $z / b$ and take the limit as $b \rightarrow \infty$, we obtain the confluent hypergeometric function $\Phi(a ; c ; z)$, i.e.

$$
\Phi(a ; c ; z)=\lim _{b \rightarrow \infty}{ }_{2} F_{1}(a, b ; c ; z / b) .
$$

In Eq. (3.12), let us replace $z$ by $z / \omega$ and take the limit as $\omega \rightarrow \infty$. Then the truncation error in the main diagonal Padé approximations to the function $\Phi(1 ; 1+\lambda ; z)$, which we shall also denote by $R_{n, n}(z)$ is given by

$$
R_{n, n}(z)=\frac{(-1)^{n} \Gamma(\lambda+1) \Gamma(n+1)}{\Gamma(2 n+\lambda+2)} \cdot \frac{z^{2 n+1} \Phi(n+1 ; 2 n+\lambda+2 ; z)}{\Psi(-n ;-2 n-\lambda ;-z)} .
$$

The function $\Psi(a ; c ; z)$ is as defined by Erdélyi [6, Chapter VI]. Since $n$ is an integer, we have [6, p. 257, Eq. (7)],

$$
\Psi(-n ;-2 n-\lambda ;-z)=\frac{\Gamma(2 n+\lambda+1)}{\Gamma(n+\lambda+1)} \Phi(-n ;-2 n-\lambda ;-z) .
$$

If we also apply Kummer's transformation [6, p. 253, Eq. (7)] to the confluent hypergeometric function appearing in the numerator of the right-hand side of equation (4.2), we find,

$$
\begin{aligned}
R_{n, n}(z)= & \frac{(-1)^{n} \Gamma(\lambda+1) \Gamma(n+1) \Gamma(n+\lambda+1)}{\Gamma(2 n+\lambda+2) \Gamma(2 n+\lambda+1)} \\
& \times \frac{z^{2 n+1} e^{z} \Phi(n+\lambda+1 ; 2 n+\lambda+2 ;-z)}{\Phi(-n ;-2 n-\lambda ;-z)} .
\end{aligned}
$$

This equation gives an exact representation of the truncation error. Before considering its asymptotic form for large $n$, it is of interest to note that in the particular case when $\lambda=0$, we can represent the error in terms of the modified spherical Bessel functions. When $\lambda=0$, the confluent hypergeometric function $\Phi(1 ; 1+\lambda ; z)$ is simply the function $e^{z}$, and we have directly from equation (4.2) that the truncation error is given by

$$
R_{n, n}(z)=(-1)^{n+1} \pi e^{z} \frac{I_{n+1 / 2}(-z / 2)}{K_{n+1 / 2}(-z / 2)} .
$$

This explicit form of the truncation error appears to have been overlooked by Luke [3], (see his Eq. (4.17)).

Returning now to the general case, we can obtain from equation (4.4), the asymptotic form of $R_{n, n}(z)$ for large $n$. Luke [3, Eq. (4.10)], has shown that 


$$
\Phi(-n ;-2 n-\lambda ;-z)=\Phi(n ; 2 n+\lambda ;-z)\left\{1+O\left(\frac{1}{n}\right)\right\} .
$$

If we now make use of the known asymptotic form of the function $\Phi(a ; c ; z)$ when both $a$ and $c$ are large and positive [6, p. 280, Eq. (17)], we have,

$$
R_{n, n}(z)=(-1)^{n} z^{2 n+1} e^{z} \frac{\Gamma(\lambda+1) \Gamma(n+1) \Gamma(n+\lambda+1)}{\Gamma(2 n+\lambda+2) \Gamma(2 n+\lambda+1)}\left\{1+O\left(\frac{1}{n}\right)\right\}
$$

which is valid for all values of $z$. Eq. (4.7) may be simplified further if we use the duplication formula for $\Gamma(2 n+\lambda+2)$ together with the fact that for large $n$,

$$
\frac{\Gamma(n+\alpha)}{\Gamma(n+\beta)}=n^{\alpha-\beta}\left\{1+O\left(\frac{1}{n}\right)\right\} \text {. }
$$

We then find

$$
R_{n, n}(z)=(-1)^{n} z^{2 n+1} e^{z} \frac{\sqrt{ } \pi \Gamma(\lambda+1)}{\sqrt{ } n 2^{2 n+\lambda+1} \Gamma(2 n+\lambda+1)}\left\{1+O\left(\frac{1}{n}\right)\right\},
$$

which agrees with a result of Luke's [3, Eq. (4.15)].

5. The Function $z^{-1} \Psi\left(1 ; 2-\omega ; z^{-1}\right)$. To conclude our analysis we shall again consider a limiting form of the hypergeometric function. If, in the function ${ }_{2} F_{1}(1, \omega ; 1+\lambda ; z)$, we replace $z$ by $-\lambda z$ and consider the limit as $\lambda \rightarrow \infty$, we obtain the function $z^{-1} \Psi\left(1 ; 2-\omega ; z^{-1}\right)$. This is a particular example of the more general result [6, p. 257, Eq. (3)],

$$
\lim _{c \rightarrow \infty}{ }_{2} F_{1}(a, b ; c ; c z)={ }_{2} F_{0}(a, b ; z)=\left(-\frac{1}{z}\right)^{a} \Psi\left(a ; a-b+1 ;-z^{-1}\right) .
$$

The function $z^{-1} \Psi\left(1 ; 2-\omega ; z^{-1}\right)$ is a many-valued function and we shall consider its principal branch, which is holomorphic in the $z$-plane cut along the negative real axis. The continued fraction representation of this function has been discussed by IVall [2], who denotes it by $\Omega(1, \omega ;-z)$.

In order to obtain an expression for the truncation error $R_{n, n}(z)$ let us again start with Eq. (3.12). On replacing $z$ by $-\lambda z$ and taking the limit as $\lambda \rightarrow \infty$, we find

$$
R_{n, n}(z)=-\omega \Gamma(n+1) \frac{\Psi\left(n+1 ; 1-\omega ; z^{-1}\right)}{\Phi\left(-n ; \omega+1 ;-z^{-1}\right)} .
$$

Again, this result is exact. In order to find an asymptotic estimate of $R_{n, n}(z)$ for large $n$, it appears more convenient first to express the $\Psi$ function as a Whittaker function (see [6, p. 264, Eq. (4) and p. 265, Eq. (8)]), and the $\Phi$ function as a generalised Laguerre polynomial [6, p. 268, Eq. (36)]. We have

$$
R_{n, n}(z)=-\frac{\Gamma(n+\omega+1)}{\Gamma(\omega)} \cdot \frac{e^{1 / 2 z} W_{-k, \omega / 2}(1 / z)}{z^{(\omega-1) / 2} L_{n}^{(\omega)}(-1 / z)},
$$

where $k=n+(\omega+1) / 2$. We can now make use of readily available results for the asymptotic form of the Whittaker function and Laguerre polynomial, to obtain the required asymptotic form for $R_{n, n}(z)$. From results given by Slater [7, Eq. 
(4.4.35)] and Szegö [8, Eq. (8.22.3), where we have replaced $n$ by $k$, we obtain after some algebra,

$$
R_{n, n}(z)=-\frac{2 \pi}{\Gamma(\omega)} \cdot \frac{e^{1 / z}}{z^{\omega}} e^{-4(k / z)}\left\{1+O\left(\frac{1}{n}\right)\right\},
$$

which is valid for $|\arg z|<\pi$. For noninteger values $\alpha$, we take $z^{\alpha}$ to be positive when $z$ is real and positive. This result agrees with that given by Luke [3, Eq. (4.26)], and again, as he has demonstrated, it can provide an excellent estimate of the error even for small values of $n$.

6. Conclusion. In this paper we have considered the problem of finding realistic a priori estimates of the truncation errors incurred when certain functions are replaced by rational approximations obtained from the main diagonal elements of the Padé table. Although an expression for these truncation errors in terms of a contour integral is readily derived, we see that a priori estimates for these errors can only be obtained if the denominator polynomials in the rational approximations are known explicitly. For certain related hypergeometric and confluent hypergeometric functions, these polynomials are known explicitly and it has been shown how realistic estimates of the truncation error may then be obtained. (For specific elementary functions which are members of this set of hypergeometric and confluent hypergeometric functions, the reader is referred to the extensive lists of such functions as given by Abramowitz and Stegun [9, Chapters 13 and 15].)

For the functions considered in this paper, we have been able to represent the truncation error explicitly in terms of the ratio of two hypergeometric (or confluent hypergeometric) functions. From these explicit results, we have then quoted the well-documented appropriate asymptotic forms of these functions in order to obtain estimates of the truncation errors in terms of elementary functions which are readily evaluated. However, this should not conceal the fact that estimates of the truncation error can be obtained directly from the contour integral expression by making use of approximate methods of evaluating integrals such as Debye's method of steepest descents. For a description of such methods, the reader is referred to de Bruijn [12].

Finally, we note that the particular asymptotic expansions for the truncation errors as given here are not the only possible ones. For example in Eq. (3.12), it might be desirable to use uniform asymptotic expansions for the hypergeometric functions occurring in this equation. Such expansions have been given by Elliott and Robinson [10], who obtained them using theorems due to Olver [11].

Acknowledgements. The author would like to thank Mr. B. Robinson for carefully checking the manuscript. This research was supported in part by a grant from the Australian Research Grants Committee.

Mathematics Department

University of Tasmania

Hobart, Tasmania

Australia 
1. E. W. Cheney \& T. H. Southard, "A survey of methods for rational approximation, with particular reference to a new method based on a formula of Darboux," SIAM Rev., v. 5, 1963, pp. 219-231. MR 28 \#1754.

2. H. S. WALL, Analytic Theory of Continued Fractions, Van Nostrand, New York, 1948. MR 10, 32 .

3. Y. L. Luke, "The Padé table and the $\tau$-method," J. Math. Phys., v. 37, 1958, pp. 110127. MR $20 \# 5558$.

4. E. LaGUERRe, "Sur le réduction en fractions continues, etc.", J. Math., 4, v. 1, 1885, pp. 135-165.

5. E. T. Whittaker \& G. N. Watson, A Course of Modern Analysis, 4th ed., Cambridge Univ. Press, New York, 1962.

6. A. Erdélyi, W. Magnus, F. Oberhettinger \& F. G. Tricomi, Higher Transcendental Functions, Vol. 1, McGraw-Hill, New York, 1953. MR 15, 419.

7. L. J. Slater, Confluent Hypergeometric Functions, Cambridge Univ. Press, New York, 1960. MR 21 \#5753.

8. G. Szegö, Orthogonal Polynomials, Amer. Math. Soc. Colloq. Publ., v. 23, Amer. Math. Soc., Providence, R. I., 1939; 2nd rev. ed., 1959. MR 1, 14; MR 21 \#5029.

9. M. Abramowitz \& I. A. Stegun, (Editors), Handbook of Mathematical Functions, with Formulas, Graphs, and Mathematical Tables, National Bureau of Standards Applied Math. Series, No. 55, U. S. Government Printing Office, Washington, D. C., 1964; reprint 1965. MR 29 \#4914; MR 31 \#1400.

10. D. Elliott \& B. Robinson, Uniform Asymptotic Estimates of the Classical Orthogonal Polynomials and some Associated Functions, Math. Dept. Tech. Rep. No. 11, University of Tasmania, 1966.

11. F. W. J. Olver, "The asymptotic solution of linear differential equations of the second order for large values of a parameter," Philos. Trans. Roy. Soc. London Ser. A, v. 247, 1954, pp. 307-327. MR 16, 695.

12. N. G. DEBruisn, Asymptotic Methods in Analysis, Bibliotheca Mathematica, v. 4, NorthHolland, Amsterdam; Noordhoff, Groningen; Interscience, New York, 1958. MR 20 \#6003. 